\title{
Repercussões fisiopatológicas, clínicas e tratamento da Encefalopatia de Wernicke:
}

\section{uma revisão}

Pathophysiological, clinical and treatment repercussions of Wernicke's Encephalopathy: a review

Repercusiones fisiopatológicas, clínicas y terapéuticas de la encefalopatía de Wernicke: una

revisión

Gustavo Soares Gomes Barros Fonseca

ORCID: https://orcid.org/0000-0002-7287-1409 Universidade CEUMA, Brasil

E-mail: gustavosgbf@gmail.com

Izabely Lima Assunção

ORCID: https://orcid.org/0000-0001-8262-9192 Universidade CEUMA, Brasil E-mail: izabelyyyyy@gmail.com

Luyla Oliveira Paes Landim Pacheco ORCID: https://orcid.org/0000-0001-8947-5851 Universidade CEUMA, Brasil

E-mail: luyla_landim@hotmail.com

Wellington Ubaldino de Freitas Júnior

ORCID: https://orcid.org/0000-0002-3513-539X Universidade CEUMA, Brasil

E-mail: wjuninho@outlook.com

Patrielly Oliveira Trindade

ORCID: https://orcid.org/0000-0002-7832-705X Universidade CEUMA, Brasil

E-mail: patriellytriindade@hotmail.com

Ademar Sodré Neto Segundo

ORCID: https://orcid.org/0000-0003-2639-3917 Universidade CEUMA, Brasil

E-mail: ademarsodre10@gmail.com

Cecília Pessoa Almeida Rego

ORCID: https://orcid.org/0000-0003-4238-3882 Universidade CEUMA, Brasil

E-mail: ceciliapessoaa@gmail.com

Janyne Daniel da Cunha França da Silva

ORCID: https://orcid.org/0000-0002-3304-9359 Universidade CEUMA, Brasil

E-mail: janyne-daniiel@live.com

Rebeca Carvalho de Aguiar

ORCID: https://orcid.org/0000-0002-0687-5620 Universidade CEUMA, Brasil

E-mail: rebeca_aguiiar10@hotmail.com

Fernando de Abreu Borges ORCID: https://orcid.org/0000-0001-8700-9240 Universidade CEUMA, Brasil

E-mail: fernandoabreuborges@gmail.com

Claudia Nery do Nascimento Coelho ORCID: https://orcid.org/0000-0002-7739-1885 Universidade CEUMA, Brasi E-mail: claudia.nnc@gmail.com

Ana Clara Nóbrega Costa

ORCID: https://orcid.org/0000-0003-4148-2379 Universidade CEUMA, Brasi

E-mail: anaclaraanobregacosta@ hotmail.com

Ana Clara Rocha Garcia

ORCID: https://orcid.org/0000-0002-5524-9829 Universidade CEUMA, Brasil

E-mail: anaaclararochatg@gmail.com

Vivianne Maria Laranjeiras Monte Freire ORCID: https://orcid.org/0000-0002-0364-9724 Universidade CEUMA, Brasil

E-mail: vivianelaranjeiiras@ hotmail.com 
Luana Caroline Oliveira Marinho

ORCID: https://orcid.org/0000-0003-3681-3225 Universidade CEUMA, Brasil

E-mail: lulimariinho02@gmail.com

Pedro de Lacerda Lima

ORCID: https://orcid.org/0000-0002-9003-2692 Universidade CEUMA, Brasil E-mail: pedroo2060@msn.com

Pietra Possapp Andrade

ORCID: https://orcid.org/0000-0002-9215-786X Universidade CEUMA, Brasil

E-mail: pietra.possaapp@ outlook.com

Ana Vitória Feitosa Barroso Maia

ORCID: https://orcid.org/0000-0002-8561-0646 Universidade CEUMA, Brasi

E-mail: vicfeitosaa@hotmail.com

Lais Lima Bonfim

ORCID: https://orcid.org/0000-0002-2816-3464 Universidade CEUMA, Brasil

E-mail: laisbonfim@hotmail.com Mikael Coutinho Silva

ORCID: https://orcid.org/0000-0003-2243-9690 Universidade CEUMA, Brasil

E-mail: mikael448@hotmail.com

Bárbara Nussrala Carvalho

ORCID: https://orcid.org/0000-0002-2678-3715 Universidade CEUMA, Brasi

E-mail: barbaranussrala@hotmail.com

Fernanda Soares Rezende Carmo

ORCID: https://orcid.org/0000-0002-9068-1553 Universidade CEUMA, Brasil

E-mail: fernanda_rezende3@hotmail.com

Vanessa Marques da Silva

ORCID: https://orcid.org/0000-0001-5638-7118

Centro Universitário da Amazônia, Brasil

E-mail: vanessamarquessdasilva@ outlook.com

David Brusaca Abreu

ORCID: https://orcid.org/0000-0001-8296-4492 Universidade CEUMA, Brasil E-mail: david.ba@gmail.com

Maria Julia Fonseca Lima Leite

ORCID: https://orcid.org/0000-0001-9489-8656 Universidade CEUMA, Brasil

E-mail: maju.fonsecaa@outlook.com

Paula Soares Machado

ORCID: https://orcid.org/0000-0001-5516-692X

Centro Universitário Christus, Brasil

E-mail: paulamachado@outlook.com

Arthur Brício Silva Costa

ORCID: https://orcid.org/0000-0003-3775-0262 Universidade CEUMA, Brasil

E-mail: arthurbricio@outlook.com

Rafael Pavão Gonçalves

ORCID: https://orcid.org/0000-0002-5234-3086 Universidade CEUMA, Brasil

E-mail: Metodologiacientifica42@gmail.com

\section{Resumo}

Dentre as encefalopatias, algumas vêm sendo mais estudadas nas últimas décadas, como a encefalopatia de Wernicke (EW), a qual é um conjunto de sinais e sintomas neuropsiquiátricos que resultam de uma deficiência nutricional de tiamina. Essa pesquisa trata-se de uma revisão bibliográfica sistemática, do tipo quantitativa, que utilizou as plataformas PubMed, Scientific Eletronic Library On-line (SciELO) e Google Scholar como base de dados para pesquisa dos artigos científicos. Foram utilizadas literaturas publicadas com recorte temporal de 2016 a 2021 , na língua inglesa que abordavam a encefalopatia de Wernicke e seus aspectos fisiopatológicos e clínicos. Constatou-se, portanto, que a deficiência da tiamina (vitamina B1) é capaz de levar o paciente a desenvolver a encefalopatia de Wernicke, quando a doença se associa à amnésia anterógrada. Ademais, a condição subjacente descrita na EW é o alcoolismo. Por conseguinte, altas doses da tiamina parenteral em pacientes de alto risco são primordiais para a melhora gradativa do paciente.

Palavras-chave: Encefalopatia de Wernicke; Tríade clássica; Alcoolismo. 


\begin{abstract}
Among the encephalopathies, some have been more studied in recent decades, such as Wernicke's encephalopathy (WE), which is a set of neuropsychiatric signs and symptoms that result from a nutritional deficiency of thiamine. This research is a systematic bibliographic review, of the quantitative type, which used the platforms PubMed, Scientific Electronic Library Online (SciELO) and Google Scholar as a database for researching scientific articles. Literature published with a time frame from 2016 to 2021, in English, that addressed Wernicke's Encephalopathy and its pathophysiological and clinical aspects were used. Therefore, it was found that the deficiency of thiamine (vitamin B1) is able to lead the patient to develop Wernicke's encephalopathy, when the disease is associated with anterograde amnesia. Furthermore, the underlying condition described in WE is alcoholism. Therefore, high doses of parenteral thiamine in high-risk patients are essential for the patient's gradual improvement.
\end{abstract}

Keywords: Wernicke's encephalopathy; Classical triad; Alcoholism.

\title{
Resumen
}

Entre las encefalopatías, algunas han sido más estudiadas en las últimas décadas, como la encefalopatía de Wernicke (EW), que es un conjunto de signos y síntomas neuropsiquiátricos que resultan de una deficiencia nutricional de tiamina. Esta investigación es una revisión bibliográfica sistemática, de tipo cuantitativo, que utilizó las plataformas PubMed, Scientific Electronic Library Online (SciELO) y Google Scholar como base de datos para la investigación de artículos científicos. Se utilizó literatura publicada con un marco temporal de 2016 a 2021, en inglés, que abordaba la Encefalopatía de Wernicke y sus aspectos fisiopatológicos y clínicos. Por tanto, se encontró que la deficiencia de tiamina (vitamina B1) es capaz de llevar al paciente a desarrollar encefalopatía de Wernicke, cuando la enfermedad se asocia con amnesia anterógrada. Además, la condición subyacente descrita en la EW es el alcoholismo. Por tanto, las dosis elevadas de tiamina parenteral en pacientes de alto riesgo son fundamentales para la mejora progresiva del paciente.

Palabras clave: Encefalopatía de Wernicke; Tríada clásica; Alcoholismo.

\section{Introdução}

As encefalopatias constituem-se de qualquer doença cerebral que cause prejuízo no funcionamento e estrutura do cérebro. A etiologia da encefalopatia é diversa: doenças isquêmicas, hipertensivas, traumas, infecções, mitocôndrias, metabólico-sistêmicas, intoxicações exógenas, neoplasias (de Almeida et al., 2021; Nascimento, 2018).

Dentre as encefalopatias, algumas vem sendo mais estudadas nas últimas décadas, como a encefalopatia de Wernicke, a qual é um conjunto de sinais e sintomas neuropsiquiátricos que resultam de uma deficiência nutricional de tiamina. Essa vitamina é necessária em todos os tecidos, sendo encontrada em altas concentrações nos músculos esqueléticos, fígado, coração, rim e cérebro (Galvão et al., 2020; de Freitas Lacerda, 2021).

A encefalopatia de Wernicke é geralmente associada ao alcoolismo, sendo caracterizada pela tríade de ataxia, paralisia parcial dos músculos oculares (oftalmoplegia) e confusão mental. Sendo assim, uma forma de dano cerebral associada ao uso indevido de álcool (Cassiano, 2020; Gomes, 2020).

O alcoolismo e alimentação desequilibrada são comuns em pacientes que desenvolvem essa síndrome, já que essa questão interferem a absorção da timina. A hiperêmese, AIDS e câncer são doenças que podem resultar da encefalopatia de Wernicke, tendo em vista a desnutrição prolongada (Galvão et al., 2020; Silva, 2020; Otárola, 2006).

O diagnóstico da encefalopatia de Wernicke é clínico. Tendo relação com a deficiência de tiamina e avaliação da desnutrição. Alguns testes devem ser solicitados para exclusão de diagnóstico, como: líquor, potenciais evocados, exames de imagem cerebral ou EEG. O diagnóstico da síndrome não é estabelecido em $25 \%$ dos casos, se os cérebros não são examinados microscopicamente (Galvão et al., 2020; Antonio et al., 2020; Zubaran et al., 1996).

O tratamento com a administração de tiamina deve ser iniciado uma vez que previne a progressão da doença e diminui a prevalência de anormalidades cerebrais. Assim, doses de 50-100mg devem ser administradas por via endovenosa por vários dias (Sotili, 2016; da SILVA et al., 2019; Zubaran et al., 1996).

O prognóstico depende da rapidez que o transtorno é tratado. Vale destacar que a perda de equilíbrio e a confusão podem persistir por meses, já a perda de memória pode ser irreversível. Já a oftalmoplegia completa bilateral e a paralisia facial são manifestações clínicas raras desta patologia. Grande parte dos pacientes desenvolvem quadros demenciais. Sem 
tratamento mais da metade dos pacientes não sobrevivem (Álvarez Hernández, 2021; Lapkaa, 2015; Kaimen-Maciel, 2008).

Nesse contexto, o presente artigo tem como objetivo realizar uma revisão sistemática com a finalidade de verificar as principais repercussões fisiopatológicas, clínicas e tratamento da encefalopatia de Wernicke, a partir de um estudo bibliográfico.

\section{Metodologia}

Trata-se de uma revisão bibliográfica sistemática, de natureza quantitativa, que utilizou as plataformas PubMed, Scientific Eletronic Library On-line (SciELO) e Google Scholar como base de dados para pesquisa dos artigos científicos. Foram utilizadas literaturas publicadas com recorte temporal de 2016 a 2021, na língua inglesa que abordavam a Encefalopatia de Wernicke e seus aspectos fisiopatológicos e clínicos.

Os descritores utilizados seguiram a descrição dos termos DeCs (Descritores em Saúde) e Medical Subject Headings (MeSH) no idioma inglês, como mostra o Quadro 1.

Quadro 1. Estratégia de busca para o estudo.

("wernicke encephalopathy"[All Fields] OR "wernicke encephalopathy"[MeSH Terms]) AND ("alcohol induced disorders, nervous system"[MeSH Terms] OR ("alcohol induced"[All Fields] AND "disorders"[All Fields] AND "nervous"[All Fields] AND "system"[All Fields]) OR "nervous system alcohol-induced disorders"[All Fields] OR ("alcohol"[All Fields] AND "induced"[All Fields] AND "disorders"[All Fields] AND "nervous"[All Fields] AND "system"[All Fields]) OR "alcohol induced disorders nervous system"[All Fields]) AND "alcoholic beverages"[MeSH Terms])

Fonte: Autores (2021).

Nesta revisão, os critérios de exclusão utilizados foram: Livros, documentos de projetos de dissertação, resumos em eventos, editoriais, revisões de literatura, relatos de caso isolados, artigos que não cumpriam os critérios de inclusão e artigos duplicados, conforme o Fluxograma 1.

Fluxograma 1 - Sistematização da filtragem de artigos para confecção do estudo.

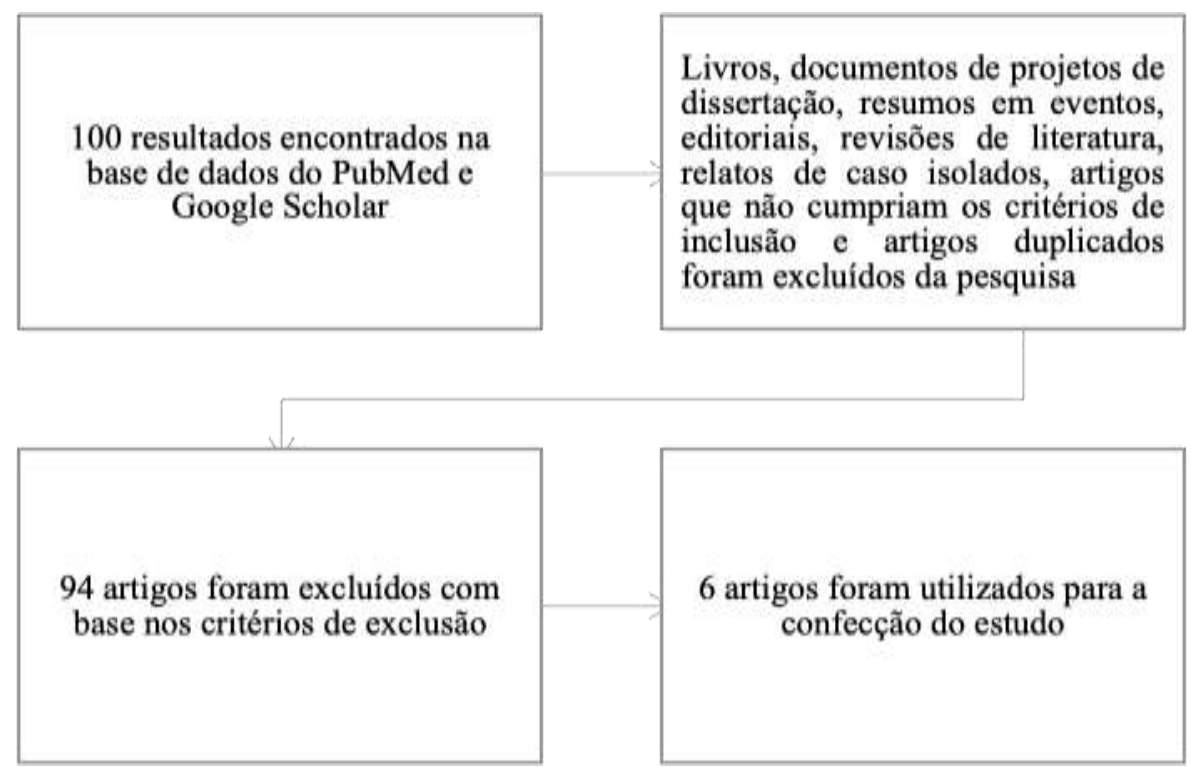

Fonte: Autores (2021). 


\section{Resultados e Discussão}

A escolha dos artigos a serem utilizados nesta revisão foi realizada por meio da leitura do título, resumo e, por fim, da leitura do artigo na íntegra, sendo realizada uma análise criteriosa dos artigos fundamentados nos critérios de inclusão e exclusão supracitados.

De acordo com o mecanismo de busca, 100 foram encontrados resultados, sendo eles na base de dados PubMed e Google Scholar, com 0 na base SciELO (Quadro 2).

Dos 100 resultados obtidos, 94 foram excluídos com base nos critérios de exclusão para a pesquisa e, além disso, não abordavam de forma objetiva sobre o tema, sendo apenas 6 artigos utilizados na confecção do estudo. Todos os artigos escolhidos serão expostos no Quadro 2, seguindo a ordem de ano de sua publicação.

Quadro 2 - Artigos selecionados nas bases de dados PubMed e Google Scholar.

\begin{tabular}{|c|c|c|}
\hline TÍTULO & AUTOR, ANO & OBSERVAÇÕES \\
\hline $\begin{array}{c}\text { Wernicke Encephalopathy-Clinical } \\
\text { Pearls }\end{array}$ & Sinha et al., 2019 & $\begin{array}{l}\text { O tratamento primário para a síndrome de Wernicke (EW) inclui } \\
\text { a administração oportuna de tiamina, para a qual a via e a } \\
\text { dosagem permanecem controversas. O julgamento clínico deve } \\
\text { ser exercido no diagnóstico e tratamento (dosagem, frequência, } \\
\text { via de administração e duração) em todos os casos de EW. } \\
\text { Ademais, o diagnóstico clínico inicial de EW é fundamental, } \\
\text { tendo em mente que a tríade clássica de sintomas muitas vezes } \\
\text { está ausente. O reconhecimento da deficiência nutricional e de } \\
\text { qualquer parte da tríade clássica deve levar ao tratamento. } \\
\text { Hipotermia, hipotensão e coma devem levantar suspeita clínica } \\
\text { para a doença. }\end{array}$ \\
\hline $\begin{array}{l}\text { Review of thiamine deficiency } \\
\text { disorders: Wernicke encephalopathy } \\
\text { and Korsakoff psychosis }\end{array}$ & $\begin{array}{l}\text { Chandrakumar et al., } \\
2019\end{array}$ & $\begin{array}{l}\text { Síndrome de Wernicke-Korsakoff (SWK) é subdiagnosticada } \\
\text { devido à apresentação clínica inconsistente e à sobreposição dos } \\
\text { sintomas com outras condições neurológicas. A identificação e o } \\
\text { tratamento individualizado da WE com base na etiologia é vital } \\
\text { para prevenir o desenvolvimento do estado amnéstico em } \\
\text { indivíduos geneticamente predispostos. }\end{array}$ \\
\hline $\begin{array}{l}\text { Wernicke encephalopathy: Guiding } \\
\text { thiamine prescription }\end{array}$ & Boulanger et al., 2016 & $\begin{array}{l}\text { A literatura indica que a tiamina deve ser prescrita em altas } \\
\text { dosagens para pacientes com diagnóstico de encefalopatia de } \\
\text { Wernicke, sendo indicadas em ambientes hospitalares e em } \\
\text { pacientes de alto risco. }\end{array}$ \\
\hline $\begin{array}{l}\text { Comprehensive review of Wernicke } \\
\text { encephalopathy: pathophysiology, } \\
\text { clinical symptoms and imaging findings }\end{array}$ & Ota et al., 2020 & $\begin{array}{l}\text { A tríade clássica de sintomas clínicos (estado mental anormal, } \\
\text { ataxia e oftalmoplegia) é encontrada em apenas 16-33\% dos } \\
\text { pacientes no exame inicial. A condição subjacente originalmente } \\
\text { descrita de WE é o alcoolismo, sendo responsável por cerca de } \\
50 \% \text { das causas de WE. }\end{array}$ \\
\hline $\begin{array}{l}\text { High-dose Parenteral Thiamine in } \\
\text { Treatment of Wernicke's } \\
\text { Encephalopathy: Case Series and } \\
\text { Review of the Literature }\end{array}$ & Nishimoto et al., 2017 & $\begin{array}{l}\text { Os pacientes com suspeita de WE foram tratados com } \geq 500 \mathrm{mg} \\
\text { de tiamina intravenosa por uma mediana de } 3 \text { dias. Assim, } 73 \% \\
\text { dos pacientes (oito em onze) apresentaram resolução dos } \\
\text { sintomas ou melhora após o tratamento. }\end{array}$ \\
\hline
\end{tabular}

Fonte: Autores (2021).

A encefalopatia de Wernicke (EW) trata-se de uma patologia grave relacionada à carência de tiamina (Vitamina B1) e associa-se geralmente ao alcoolismo. O seu quadro clínico é caracterizado pela tríade clássica de ataxia, oftalmoplegia e confusão mental. Frente à análise bibliográfica, observou-se algumas controvérsias entre literaturas, sendo importante destacálas e discuti-las.

Foi observado que, por mais que o tratamento primário para a EW seja a administração de tiamina, a sua via e dosagem permanecem controversas, sendo necessário um bom diagnóstico, visto que a tríade clássica muitas vezes encontra-se 
ausente, mas sintomas como hipotermia, hipotensão e coma levantam a suspeita clínica para a encefalopatia de Wernicke (Sinha et al., 2019).

De acordo com Chandrakumar et al. (2019), a apresentação clínica da encefalopatia de Wernicke (ou síndrome de Wernicke-Korsakoff, quando a encefalopatia é associada à amnésia anterógrada) é inconsistente, levando muitas vezes a um diagnóstico errôneo, o que pode ser um problema grave devido à sua alta morbimortalidade em certas situações. Ademais, fazse necessário identificar de forma eficiente e tratar os pacientes com base na etiologia, visto que muitos são geneticamente predispostos a desenvolver amnésia pela deficiência da tiamina.

Já Boulanger et al. (2016) afirmam que altas doses parenterais de tiamina no âmbito hospitalar para pacientes portadores de encefalopatia de Wernicke que se encontram em alto risco é primordial e possui eficácia comprovada. Segundo o estudo, a posologia recomendada é de $250 \mathrm{mg}$, diariamente, por 3 a 5 dias, que pode ser aumentada para $500 \mathrm{mg}, 3 \mathrm{vezes}$ ao dia, e uma dose adicional de $250 \mathrm{mg}$ por mais 3 a 5 dias. Deste modo, o quadro clínico grave regride à medida em que a vitamina B1 é administrada no paciente com EW.

Como foi visto por Sinha et al. (2019), Ota et al. (2020), em seus estudos, também mostraram que a tríade clássica da encefalopatia de Wernicke (estado mental anormal, ataxia e oftalmoplegia) pode se encontrar ausente em diversos casos da doença. Foi visto que, no exame inicial, ela somente foi encontrada em 16 a $33 \%$ dos pacientes, mais uma vez reafirmando a inespecificidade do quadro clínico da EW. Ademais, foi comprovado que a principal etiologia é o alcoolismo, responsável por cerca de metade dos casos.

Ao seguir a mesma linha de raciocínio de Boulanger et al. (2016), Nishimoto et al. (2017), em seu estudo, também afirmam que pacientes com suspeita ou com diagnóstico confirmado de encefalopatia de Wernicke foram tratados com altas doses parenterais de tiamina. Foram administradas doses $\geq 500 \mathrm{mg}$ de tiamina intravenosa por uma mediana de 3 dias. Dessa forma, 8 dos 11 pacientes (73\%) apresentaram resolução dos sintomas ou melhora após o tratamento, ou seja, com a dose adicional referida por Boulanger et al. (2016), supõe-se que os outros pacientes também apresentariam melhora gradativa, comprovando a eficácia posológica do protocolo ofertado de tiamina parenteral.

\section{Conclusão}

De acordo com as literaturas analisadas, tornou-se perceptível que a deficiência da tiamina (vitamina B1) é capaz de levar o paciente a desenvolver a encefalopatia de Wernicke, ou síndrome de Wernicke-Korsakoff, quando a doença se associa à amnésia anterógrada. Muitas vezes o diagnóstico é inespecífico e a tríade clássica é ausente, devendo-se ter um bom raciocínio clínico para diagnosticar a doença e tratar corretamente o paciente com a dose correta de tiamina. Ademais, altas doses da tiamina parenteral em pacientes de alto risco são primordiais para a melhora gradativa do paciente, de 3 a 5 dias com uma dose inicial de $250 \mathrm{mg}$, que pode se estender até $500 \mathrm{mg}$.

Além disso, pode-se perceber que o conhecimento, a antecipação e o reconhecimento da doença podem levar o paciente a um melhor prognóstico, sendo fundamental a abordagem multiprofissional para melhor conduta. Nota-se, dessa forma, a necessidade do desenvolvimento de novos estudos, sobretudo meta-análises e ensaios clínicos a respeito da melhor dose para pacientes portadores dessa encefalopatia para que, dessa forma, a patologia seja melhor elucidada.

\section{Referências}

Álvarez Hernández, N. (2021). Revisión de pacientes con sospecha de síndrome de Wernicke-Korsakoff en el servicio de urgencias.

Antonio, A. C. P., Cigolini, M. P., Secchi, T. L., \& Mello, J. M. D. R. B. D. (2020). Coma irreversível em paciente portadora de esquizofrenia paranóide: encefalopatia de Wernicke após síndrome de realimentação: um relato de caso. Clinical and biomedical research. Porto Alegre. vol. 40 , no. 4 (2020), p. $242-$ 246.

Boulanger, A. S., Paquette, I., Létourneau, G., \& Richard-Devantoy, S. (2016). Wernicke encephalopathy: Guiding thiamine prescription. L'encephale, 43(3), 259-267. 
Research, Society and Development, v. 10, n. 16, e580101623857, 2021

Cassiano, L. M. G. (2020). Neuroinflamação, metabolismo energético e reprogramação epigenética na neurorregeneração hipocampal em modelo ex vivo de deficiência de tiamina.

Chandrakumar, A., Bhardwaj, A., \& W`t Jong, G. (2019). Review of thiamine deficiency disorders: Wernicke encephalopathy and Korsakoff psychosis. Journal of basic and clinical physiology and pharmacology, 30(2), 153-162.

Silva, G. S., Bonini, V. C. R., Chiqueto, S. G. R., \& dos Santos, C. N. I. (2019). Tratamento da síndrome de wernicke-korsakof: reposição e controle de tiamina. anais do fórum de iniciação científica do unifunec, 10(10).

Almeida, G. F., Xavier, G. M. C. A., Rodrigues, R. M. G., Monteiro, R. B. G., Pessoa, M. L. A., Abrantes, J. J. A., ... \& Lessa, N. M. (2021). Repercussões fisiopatológicas e clínicas da encefalopatia de Hashimoto: revisão de literatura. Research, Society and Development, 10 (12), e424101220669-e424101220669.

Freitas Lacerda, R., \& Romano, I. C. S. (2021). A deficiência de tiamina pode ajudar na compreensão dos mecanismos neurodegenerativos?. South American Journal of Basic Education, Technical and Technological, 8(2), 932-939.

Galvão, N. D., Reolon, I. M., Hércules, M. B., Arruda, A. G. M., Santos, M. E. C., Teixeira, M. C., ... \& de Souza Lima, M. (2020). Síndrome de WernickeKorsakoff associada ao álcool. Brazilian Journal of Health Review, 3(6), 18435-18444.

Gomes, K. C. (2020). Estratégias terapêuticas para a deficiência de Tiamina.

Kaimen-Maciel, D. R., Rocha, F. H., \& Alexandre, D. (2008). Encefalopatia de Wernicke: Manifestação clínica inicial atípica. Rev Neurocienc, 1, 1-3.

Lapkaa, Y. S., \& Llorensb, M. (2015). Encefalopatía Wernicke-Korsakoff: Revisión de un caso clínico. Revista de Patología Dual, 2 (4), 24.

Nascimento, L. E. (2018). Santos. Estudo por imagem das encefalopatias e neurocisticercose em Hospital Público no município de Santos-SP, Brasil. UNILUS Ensino e Pesquisa, 15(39)

Nishimoto, A., Usery, J., Winton, J. C., \& Twilla, J. (2017). High-dose parenteral thiamine in treatment of Wernicke's encephalopathy: case series and review of the literature. in vivo, 31(1), 121-124.

Ota, Y., Capizzano, A. A., Moritani, T., Naganawa, S., Kurokawa, R., \& Srinivasan, A. (2020). Comprehensive review of Wernicke encephalopathy: pathophysiology, clinical symptoms and imaging findings. Japanese journal of radiology, 1-12.

Otárola U, C., Briceño F, J., Reyes S, E., Contreras R, M. D. L. A., \& Guirao B, P. (2006). Encefalopatía de Wernicke. Bol. Hosp. San Juan de Dios, $284-289$.

Silva, M. C. (2020). Alcoolismo e deficiências nutricionais (Bachelor's thesis, [sn]).

Sinha, S., Kataria, A., Kolla, B. P., Thusius, N., \& Loukianova, L. L. (2019, June). Wernicke encephalopathy—clinical pearls. In Mayo Clinic Proceedings (Vol. 94, No. 6, pp. 1065-1072). Elsevier.

Sotili, M. (2016). Abuso de álcool e síndrome de Wernicke-Korsakoff: repercussões cognitivas e na qualidade de vida dos familiares de alcoolistas.

Zubaran, C., Fernandes, J., Martins, F., Souza, J., Machado, R., \& Cadore, M. (1996). Clinical and neurophatological aspects of Wernicke-Korsakoff syndrome. Revista de saude publica, 30(6), 602-608. 